Bull. Korean Math. Soc. 46 (2009), No. 3, pp. 435-437

DOI 10.4134/BKMS.2009.46.3.435

\title{
JORDAN $\theta$-DERIVATIONS ON LIE TRIPLE SYSTEMS
}

\author{
AbBas NaJATi
}

ABstract. In this paper we prove that every Jordan $\theta$-derivation on a Lie triple system is a $\theta$-derivation. Specially, we conclude that every Jordan derivation on a Lie triple system is a derivation.

\section{Introduction}

The concept of Lie triple system was first introduced by N. Jacobson $[2,3]$ (see also [4]). We recall that a Lie triple system is a vector space $\mathcal{J}$ over a field $\mathbb{K}$ with a trilinear mapping $\mathcal{J} \times \mathcal{J} \times \mathcal{J} \ni(x, y, z) \mapsto[x, y, z] \in \mathcal{J}$ satisfying the following axioms

(i) $[x, y, z]=-[y, x, z]$,

(ii) $[x, y, z]+[y, z, x]+[z, x, y]=0$,

(iii) $[u, v,[x, y, z]]=[[u, v, x], y, z]+[x,[u, v, y], z]+[x, y,[u, v, z]]$

for all $u, v, x, y, z \in \mathcal{J}$. It follows from (i) that $[x, x, y]=0$ for all $x, y \in \mathcal{J}$.

It is clear that every Lie algebra with product [.,.] is a Lie triple system with respect to $[x, y, z]:=[[x, y], z]$. Conversely, any Lie triple system $\mathcal{J}$ can be considered as a subspace of a Lie algebra (Bertram [1], Jacobson [3]).

Throughout this paper, let $\mathbb{C}$ be the complex filed and $\mathcal{J}$ be a Lie triple system over $\mathbb{C}$.

Definition 1.1. Let $\theta: \mathcal{J} \rightarrow \mathcal{J}$ be a $\mathbb{C}$-linear mapping. A $\mathbb{C}$-linear mapping $D: \mathcal{J} \rightarrow \mathcal{J}$ is called a $\theta$-derivation on $\mathcal{J}$ if

$$
D([x, y, z])=[D(x), \theta(y), \theta(z)]+[\theta(x), D(y), \theta(z)]+[\theta(x), \theta(y), D(z)]
$$

for all $x, y, z \in \mathcal{J}$. If $\theta=I_{\mathcal{J}}$, a $\theta$-derivation is called a derivation.

Let $u, v \in \mathcal{J}$ and $D_{u, v}: \mathcal{J} \rightarrow \mathcal{J}$ be a mapping defined by

$$
D_{u, v}(x):=[u, v, x]
$$

for all $x \in \mathcal{J}$. It is clear that $D_{u, v}$ is $\mathbb{C}$-linear and we get from (iii) that the mapping $D_{u, v}$ is a derivation on $\mathcal{J}$.

Received March 27, 2008.

2000 Mathematics Subject Classification. 16W25, 17A40.

Key words and phrases. Lie triple system, $\theta$-derivation, Jordan $\theta$-derivation.

(C)2009 The Korean Mathematical Society 
Definition 1.2. Let $\theta: \mathcal{J} \rightarrow \mathcal{J}$ be a $\mathbb{C}$-linear mapping. A $\mathbb{C}$-linear mapping $D: \mathcal{J} \rightarrow \mathcal{J}$ is called a Jordan $\theta$-derivation on $\mathcal{J}$ if

$$
D([x, y, x])=[D(x), \theta(y), \theta(x)]+[\theta(x), D(y), \theta(x)]+[\theta(x), \theta(y), D(x)]
$$

for all $x, y \in \mathcal{J}$. If $\theta=I_{\mathcal{J}}$, a Jordan $\theta$-derivation is called a Jordan derivation.

In [5], M. Sal Moslehian and Th. M. Rassias have studied the stability of derivations in normed Lie triple systems associated with a Cauchy-Jensen additive mapping.

\section{Main results}

It is clear that every $\theta$-derivation on a Lie triple system $\mathcal{J}$ is a Jordan $\theta$ derivation. In this section we prove that every Jordan $\theta$-derivation on a Lie triple system $\mathcal{J}$ is a $\theta$-derivation. So we conclude that every Jordan derivation on $\mathcal{J}$ is a derivation.

Throughout this section $D, \theta: \mathcal{J} \rightarrow \mathcal{J}$ are $\mathbb{C}$-linear mappings and $A_{D, \theta}:$ $\mathcal{J} \times \mathcal{J} \times \mathcal{J} \rightarrow \mathcal{J}$ is a mapping defined by

$$
A_{D, \theta}(x, y, z):=[D(x), \theta(y), \theta(z)]+[\theta(x), D(y), \theta(z)]+[\theta(x), \theta(y), D(z)]
$$

for all $x, y, z \in \mathcal{J}$. It is clear that the mapping $A_{D, \theta}$ is trilinear and $A_{D, \theta}(x, x$, $y)=0$ for all $x, y \in \mathcal{J}$.

Theorem 2.1. Let $D: \mathcal{J} \rightarrow \mathcal{J}$ be a Jordan $\theta$-derivation. Then $D$ is a $\theta$ derivation.

Proof. Since $D: \mathcal{J} \rightarrow \mathcal{J}$ is a Jordan $\theta$-derivation, $A_{D, \theta}(x, y, x)=D([x, y, x])$ for all $x, y \in \mathcal{J}$. Therefore we have

$$
\begin{aligned}
D([x+z, y, x+z])= & {[D(x)+D(z), \theta(y), \theta(x)+\theta(z)] } \\
& +[\theta(x)+\theta(z), D(y), \theta(x)+\theta(z)] \\
& +[\theta(x)+\theta(z), \theta(y), D(x)+D(z)] \\
= & D([x, y, x])+D([z, y, z]) \\
& +A_{D, \theta}(x, y, z)+A_{D, \theta}(z, y, x)
\end{aligned}
$$

for all $x, y, z \in \mathcal{J}$. On the other hand, we have

$$
[x+z, y, x+z]=[x, y, x]+[z, y, z]+[x, y, z]+[z, y, x]
$$

for all $x, y, z \in \mathcal{J}$. Therefore

$$
\begin{aligned}
D([x+z, y, x+z])= & D([x, y, x])+D([z, y, z]) \\
& +D([x, y, z])+D([z, y, x])
\end{aligned}
$$

for all $x, y, z \in \mathcal{J}$. It follows from (2.1) and (2.2) that

$$
D([x, y, z])+D([z, y, x])=A_{D, \theta}(x, y, z)+A_{D, \theta}(z, y, x)
$$

for all $x, y, z \in \mathcal{J}$. Since $[z, y, x]=[x, y, z]-[x, z, y]$, we get that

$$
D([x, y, z])+D([z, y, x])=2 D([x, y, z])-D([x, z, y])
$$


for all $x, y, z \in \mathcal{J}$. Also

$$
\begin{aligned}
& A_{D, \theta}(x, y, z)-A_{D, \theta}(x, z, y) \\
= & {[D(x), \theta(y), \theta(z)]+[\theta(x), D(y), \theta(z)]+[\theta(x), \theta(y), D(z)] } \\
& \quad-[D(x), \theta(z), \theta(y)]-[\theta(x), D(z), \theta(y)]-[\theta(x), \theta(z), D(y)] \\
= & ([D(x), \theta(y), \theta(z)]+[\theta(z), D(x), \theta(y)]) \\
& \quad+([\theta(x), D(y), \theta(z)]+[\theta(z), \theta(x), D(y)]) \\
& \quad+([\theta(x), \theta(y), D(z)]+[D(z), \theta(x), \theta(y)]) \\
= & {[\theta(z), \theta(y), D(x)]+[\theta(z), D(y), \theta(x)]+[D(z), \theta(y), \theta(x)] } \\
= & A_{D, \theta}(z, y, x)
\end{aligned}
$$

for all $x, y, z \in \mathcal{J}$. So

$$
A_{D, \theta}(x, y, z)+A_{D, \theta}(z, y, x)=2 A_{D, \theta}(x, y, z)-A_{D, \theta}(x, z, y)
$$

for all $x, y, z \in \mathcal{J}$. We get from (2.3), (2.4) and (2.5) that

$$
2 D([x, y, z])-D([x, z, y])=2 A_{D, \theta}(x, y, z)-A_{D, \theta}(x, z, y)
$$

for all $x, y, z \in \mathcal{J}$. Letting $y=z$ in $(2.6)$, we get $D([x, y, y])=A_{D, \theta}(x, y, y)$ for all $x, y \in \mathcal{J}$. Since $D([x, y+z, y+z])=A_{D, \theta}(x, y+z, y+z)$ and $[., . .],. A_{D, \theta}$ are trilinear, we have

$$
D([x, y, z])+D([x, z, y])=A_{D, \theta}(x, y, z)+A_{D, \theta}(x, z, y)
$$

for all $x, y, z \in \mathcal{J}$. Adding (2.6) to (2.7), we infer that $D([x, y, z])=A_{D, \theta}(x, y, z)$ for all $x, y, z \in \mathcal{J}$. So the proof is completed.

Corollary 2.2. Every Jordan derivation on a Lie triple system is a derivation.

\section{References}

[1] W. Bertram, The Geometry of Jordan and Lie Structures, Lecture Notes in Math., Vol. 1754, Springer-Verlag, 2000.

[2] N. Jacobson, Lie and Jordan triple systems, Amer. J. Math. 71 (1949), 149-170.

[3] , General representation theory of Jordan algebras, Trans. Amer. Math. Soc. 70 (1951), 509-530.

[4] W. G. Lister, A structure theory of Lie triple systems, Trans. Amer. Math. Soc. 72 (1952), $217-242$.

[5] M. S. Moslehian and Th. M. Rassias, Generalized Hyers-Ulam stability of mappings on normed Lie triple systems, Math. Inequal. Appl. 11 (2008), no. 2, 371-380.

Department of Mathematics

FACULTY OF SCIENCES

University of Mohaghegh Ardabili

ARDABIL, IRAN

E-mail address: a.nejati@yahoo.com 